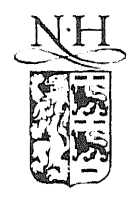

HISSFVIFR

\title{
The iterative solution of fully implicit discretizations of three-dimensional transport models
}

\author{
P.J. van der Houwen*, B.P. Sommeijer, J. Kok \\ (WI, P.O. Box 94079, 1090 GB Amsterdam. The Netherlands
}

\begin{abstract}
We investigate the use of approximate factorization and diagonalizing techniques for solving iteratively fully implicit numerical models of three-dimensional transport-chemistry problems. In particular, we investigate various possibilities that can take advantage of the parallelization and vectorization facilities offered by parallel vector computers. 1997 Elsevier Science B.V.
\end{abstract}

Keywords: Advection-diffusion-reaction equations; Splitting methods; Runge-Kutta methods

\section{Introduction}

The mathematical model describing transport processes of salinity, pollutants, etc., combined with their bio-chemical interactions, is defined by an initial-boundary value problem for the system of $3 \mathrm{D}$ advection-diffusion-reaction equations

$$
\begin{aligned}
\frac{\partial c_{\mu}}{\partial t}=L(u, v, w) r_{\mu}+g_{\mu}\left(x, y, z, t, c_{1}, \ldots, c_{m}\right), \quad \mu=1, \ldots, m, & \\
L(u, v, w) c_{\mu}:= & -u \frac{\partial}{\partial x} c_{\mu}-v \frac{\partial}{\partial y} c_{\mu}-w \frac{\partial}{\partial z} c_{\mu}+\frac{\partial}{\partial x}\left(\varepsilon_{r} \frac{\partial c_{\mu}}{\partial x}\right) \\
& +\frac{\partial}{\partial y}\left(\varepsilon_{y} \frac{\partial c_{\mu}}{\partial y}\right)+\frac{\partial}{\partial z}\left(\varepsilon_{z} \frac{\partial c_{\mu}}{\partial z}\right) .
\end{aligned}
$$

Here, the various quantities are defined as follows:

$r_{\mu}$ : concentrations of the contaminants,

$u, v, w: \quad$ local fluid velocities in $x, y, z$ directions (assumed to be divergence free),

\footnotetext{
The investigations reported in this paper were partly supported by the Dutch HPCN Program.

* Corresponding author.
} 
$\varepsilon_{x}, \varepsilon_{y}, \varepsilon_{z}:$ diffusion coefficients in $x, y, z$ directions (may depend on the species),

$g_{\mu}:$ reaction terms (e.g., chemical interactions) and emissions from sources.

The velocities $u, v, w$, and diffusion coefficients $\varepsilon_{x}, \varepsilon_{y}, \varepsilon_{z}$ are assumed to be known in advance. The terms $g_{\mu}$ describe chemical reactions, emissions from sources, etc., and therefore depend on the concentrations. The mutual coupling of the equations in the system (1.1) is due to the functions $g_{\mu}$. The boundary conditions are either of Dirichlet type or of Neumann type.

Along the lines described in $[4,6]$, we replace in (1.1) the physical domain by a set of $N$ Cartesian grid points with mesh sizes $\Delta x, \Delta y$, and $\Delta z$, the advection terms by upwind-biased $\kappa=1 / 3$ discretizations (see [8]), and the diffusion terms by symmetric three-point discretizations. This results in a semidiscrete, $m N$-dimensional initial value problem (IVP)

$$
\frac{\mathrm{d} \boldsymbol{C}(t)}{\mathrm{d} t}=\boldsymbol{F}(t, \boldsymbol{C}(t)):=\boldsymbol{H}(t, \boldsymbol{C}(t))+\boldsymbol{G}(t, \boldsymbol{C}(t)), \quad \boldsymbol{C}\left(t_{0}\right)=\boldsymbol{C}_{0} .
$$

Here $C$ contains the $m$ concentrations $c_{\mu}$ at all $N$ grid points, $C_{0}$ defines the initial values, $\boldsymbol{H}(t, \boldsymbol{C}(t))$ represents the advection-diffusion terms, and $\boldsymbol{G}(t, \boldsymbol{C}(t))$ contains the reaction terms and emissions from sources. $\boldsymbol{H}(t, \boldsymbol{C})$ is linear in $\boldsymbol{C}$ with a block-diagonal matrix of coefficients. The $m$ diagonal blocks have dimension $N$ and contain (at most) only 13 nonzero diagonals (see also Section 2.1). We remark that these diagonal blocks are all the same if we use the same boundary conditions and diffusion coefficients for the various species.

From the definition of the operator $L$ it follows that $\boldsymbol{H}(t, \boldsymbol{C})$ can be split into three terms corresponding with the derivatives with respect to $x, y$ and $z$, respectively (dimensional splitting). Hence, $\boldsymbol{H}(t, C)$ can be written as

$$
\boldsymbol{H}(t, \boldsymbol{C})=(X(t)+Y(t)+Z(t)) \boldsymbol{C},
$$

where the matrices $X, Y$ and $Z$ are again block-diagonal. Each of these blocks contains 5 nonzero diagonals. Moreover, all $m$ blocks belonging to $X$ (or $Y$, or $Z$ ) are identical if we use the same boundary conditions and diffusion coefficients for the various species.

In general, $G(t, C)$ is nonlinear in $\boldsymbol{C}$, but at each grid point, it only depends on the $m$ concentrations $c_{\mu}$ at that particular grid point. The reaction term $G$ may be considered as nonstiff, because the chemistry in shallow water transport problems usually has large time constants. However, the advection-diffusion term $H$ introduces stiffness, due to the relatively small vertical mesh size $\Delta z$.

In order to cope with the stiffness of the IVP (1.2), we shall use for the time discretization an implicit discretization formula. Since transport problems usually are advection dominated, this implicit formula should at least be A-stable and preferably L-stable. The choice of such a highly stable time discretization formula now depends on the required order of time accuracy. If second-order accuracy suffices, one may use in the first integration step the (selfstarting) first-order, L-stable backward Euler method, and in all subsequent steps, the second-order, L-stable backward differentiation formula (BDF). Thus,

$$
R\left(t_{n+1}, C_{n+1}\right)=0,
$$

where

$$
\begin{array}{ll}
\boldsymbol{R}\left(t_{1}, \boldsymbol{C}\right):=C-\Delta t \boldsymbol{F}\left(t_{1}, \boldsymbol{C}\right)-\boldsymbol{C}_{0}, & n=0, \\
\boldsymbol{R}\left(t_{n+1}, \boldsymbol{C}\right):=\boldsymbol{C}-\frac{2}{3} \Delta t \boldsymbol{F}\left(t_{n+1}, C\right)-\frac{1}{3}\left[4 C_{n}-C_{n-1}\right], & n \geqslant 1 .
\end{array}
$$


If third-order time accuracy is desired, we may use the (selfstarting) L-stable, two-stage Radau IIA discretization

$$
\boldsymbol{R}_{1}\left(t_{n+1 / 3}, t_{n+1}, \boldsymbol{A}_{n+1 / 3}, \boldsymbol{C}_{n+1}\right)=0, \quad \boldsymbol{R}_{2}\left(t_{n+1 / 3}, t_{n+1}, \boldsymbol{A}_{n+1 / 3}, C_{n+1}\right)=0,
$$

where $\boldsymbol{A}_{n+1 / 3}$ may be considered as an auxiliary vector and where the residual functions $\boldsymbol{R}_{1}$ and $\boldsymbol{R}_{2}$ are defined by

$$
\begin{aligned}
& \boldsymbol{R}_{1}\left(t_{n+1 / 3}, t_{n+1}, \boldsymbol{A}, \boldsymbol{C}\right):=\boldsymbol{A}-\frac{5}{12} \Delta t \boldsymbol{F}\left(t_{n+1 / 3}, \boldsymbol{A}\right)+\frac{1}{12} \Delta t \boldsymbol{F}\left(t_{n+1}, \boldsymbol{C}\right)-\boldsymbol{C}_{n}, \\
& \boldsymbol{R}_{2}\left(t_{n+1 / 3}, t_{n+1}, \boldsymbol{A}, \boldsymbol{C}\right):=\boldsymbol{C}-\frac{3}{4} \Delta t \boldsymbol{F}\left(t_{n+1 / 3}, \boldsymbol{A}\right)-\frac{1}{4} \Delta t \boldsymbol{F}\left(t_{n+1}, \boldsymbol{C}\right)-\boldsymbol{C}_{n}
\end{aligned}
$$

The aim of this paper is to develop efficient iterative methods for solving the systems (1.3) and (1.4).

\section{The iteration process}

In order to solve the nonlinear systems (1.3) or (1.4) there are two often used approaches: fixed-point iteration and modified Newton iteration. Let us first consider the fixed-point iteration process for the BDF discretization (1.3)

$$
\boldsymbol{C}^{(\nu)}=\boldsymbol{C}^{(\nu-1)}-\boldsymbol{R}\left(t_{n+1}, \boldsymbol{C}^{(\nu-1)}\right), \quad \nu=1,2, \ldots
$$

This iteration process is relatively cheap, highly vectorizable, and highly parallelizable. However, it will only converge if $\Delta t$ is extremely small. To make this more precise, we ignore the nonstiff chemistry and the usually small diffusion terms. Then, fixed-point iteration will converge if the CFL number $Q$, defined as

$$
Q:=\Delta t\left(\frac{|u|}{\Delta x}+\frac{|v|}{\Delta y}+\frac{|w|}{\Delta z}\right)
$$

is sufficiently small. Due to the usually small mesh size $\Delta z$, such a convergence condition imposes a severe restriction on the time step $\Delta t$, unless $w=0$. For example, in practical situations $|w| / \Delta z$ can be as large as $0.1 \mathrm{sec}^{-1}$, forcing this process to take time steps less than 10 seconds to achieve, say $Q \leqslant 1$ (the horizontal advection terms are less dangerous, because the horizontal mesh sizes usually are a factor 100 or 1000 larger). Evidently, we have to discard the fixed-point iteration process.

At the other end of the scale, we can generate successive approximations $\boldsymbol{C}^{(\nu)}$ to $\boldsymbol{C}_{n+1}$ by means of the modified Newton process. Let us again consider the BDF discretization (1.3) for which modified Newton reads

$$
\boldsymbol{C}^{(\nu)}=\boldsymbol{C}^{(\nu-1)}-J^{-1} \boldsymbol{R}\left(t_{n+1}, \boldsymbol{C}^{(\nu-1)}\right), \quad J:=I-\alpha \Delta t \frac{\partial \boldsymbol{F}}{\partial \boldsymbol{C}}, \quad \nu=1,2, \ldots,
$$

where $\partial \boldsymbol{F} / \partial \boldsymbol{C}=\partial(\boldsymbol{H}+\boldsymbol{G}) / \partial \boldsymbol{C}$ is the Jacobian and where $\alpha=1$ for $n=0$ and $\alpha=2 / 3$ for $n \geqslant 1$. Each Newton iteration requires the solution of a linear system with $J$ as its matrix of coefficients. In the case of linear interaction terms, only one Newton iteration suffices, because the advection-diffusion terms are also linear. But also in the case of nonlinear interaction, the Newton process is expected to converge under rather mild conditions on the time step $\Delta t$. However, due to the different coupling of the unknowns in the functions $\boldsymbol{H}$ and $\boldsymbol{G}$, and due to the fact that we are dealing with three spatial dimensions, the linear algebra involved in solving the linear Newton systems is extremely expensive, so that we also have to drop the modified Newton algorithm. 
In this paper, we shall describe an iteration scheme that is in some sense a compromise between the two extreme cases of fixed-point iteration and modified Newton iteration. This iteration scheme will be separately discussed for the BDF discretization (1.3) and the Radau discretization (1.4).

\subsection{The BDF discretization}

By observing that the modified Newton process (2.3) can be interpreted as fixed-point iteration in which the residual term $\boldsymbol{R}\left(t_{n+1}, C^{(\nu-1)}\right)$ is preconditioned by the matrix $J$, we are led to define for (1.3) a preconditioned fixed-point iteration process of the form

$$
\boldsymbol{C}^{(\nu)}=\boldsymbol{C}^{(\nu-1)}-\tilde{J}^{-1} \boldsymbol{R}\left(t_{n+1}, \boldsymbol{C}^{(\nu-1)}\right), \quad \nu=1,2, \ldots,
$$

where the matrix $\tilde{J}^{-1}$ should remove the stiffness from the residual term. Evidently, any nonsingular matrix $\tilde{J}$ defines a consistent iteration scheme, that is, if the iteration scheme converges, then it converges to the solution of (1.3). The matrix $\tilde{J}$ may also be interpreted as a smoothing matrix which removes the high frequencies from the residual term.

Our first concern is to choose $\tilde{J}$ such that we have convergence as $\Delta z \rightarrow 0$. Suppose that we define $\tilde{J}$ by the "vertical-preconditioning" matrix $\tilde{J}=I-\alpha \Delta t Z$, where $Z$ is the matrix occurring in (1.2b). Then, (2.4) takes the form

$$
(I-\alpha \Delta t Z)\left(C^{(\nu)}-C^{(\nu-1)}\right)=-R\left(t_{n+1}, C^{(\nu-1)}\right), \quad \nu=1,2, \ldots
$$

Since the matrix $Z$ only represents the vertical coupling in the advection diffusion terms, the linear system $\left(2.4^{\prime}\right)$ can be decoupled into $N_{x y}$ "vertical" linear pentadiagonal subsystems, where $N_{x y}$ is the number of grid points in the horizontal plane. Therefore, the solution of the system $\left(2.4^{\prime}\right)$ has a considerable amount of intrinsic parallelism and vectorization, and can be done extremely fast on tarallel vector computers like Cray architectures.

Although the vertical advection (and diffusion) terms are the most dangerous ones, it is not recomiendable to forget about the horizontal advection (and diffusion) terms. Therefore, we shall apply a similar "horizontal" preconditioning of the residual term. This results into the iteration process

$$
\begin{aligned}
& (I-\alpha \Delta t X)(I-\alpha \Delta t Y)(I-\alpha \Delta t Z)\left(C^{(\nu)}-C^{(\nu-1)}\right)=-\boldsymbol{R}\left(t_{n+1}, \boldsymbol{C}^{(\nu-1)}\right), \\
& \quad \nu=1,2, \ldots
\end{aligned}
$$

Each iteration requires the sequential solution of three linear systems, but by virtue of the structure of the matrices $X, Y$ and $Z$ (cf. (1.2b)), these systems can be decoupled respectively into $N_{x y}, N_{x z}$ and $N_{y z}$ linear pentadiagonal subsystems (compare the decoupling in $\left(2.4^{\prime}\right)$ ). Here, $N_{y z}$ and $N_{x z}$ are defined in a similar way as $N_{x y}$ and denote the numbers of gridpoints in the vertical "north-south" and "east-west" planes.

The process (2.5) may be considered as the method of Approximate Factorizations applied to the modified Newton process (2.3) (cf. [3, p. 439]). This approach replaces the expensive linear system in (2.3) by the set of pentadiagonal subsystems involved in (2.5).

For future reference, it is convenient to give an explicit expression for the matrices $X, Y$ and $Z$ occurring in (2.5). For simplicity, we present this expression for constant diffusion coefficients $\varepsilon_{x}$, 
$\varepsilon_{y}$ and $\varepsilon_{z}$ and constant (positive) velocities $u, v$ and $w$ (in an actual implementation of numerical transport models, one should use more complicated discretization formulas which also preserve the conservation of mass in the case of nonconstant velocities). Taking $\varepsilon_{x}, \varepsilon_{y}, \varepsilon_{z}, u, v$ and $w$ constant, we obtain at a particular grid point $P_{i j k}:=(i \Delta x, j \Delta y, k \Delta z)$ the difference formula

$$
\boldsymbol{H}_{i j k}=(X \boldsymbol{C})_{i j k}+(Y C)_{i j k}+(Z C)_{i j k}
$$

where

$$
\begin{aligned}
(X C)_{i j k} & :=-\left(3 B_{1}+2 D_{1}\right) c_{i, j, k}-\left(2 B_{1}-D_{1}\right) c_{i+1, j, k}+\left(6 B_{1}+D_{1}\right) c_{i-1, j, k}-B_{1} c_{i-2, j, k} \\
(Y C)_{i j k} & :=-\left(3 B_{2}+2 D_{2}\right) c_{i, j, k}-\left(2 B_{2}-D_{2}\right) c_{i, j+1, k}+\left(6 B_{2}+D_{2}\right) c_{i, j-1, k}-B_{2} c_{i, j-2, k}, \\
(Z C)_{i j k} & :=-\left(3 B_{3}+2 D_{3}\right) c_{i, j, k}-\left(2 B_{3}-D_{3}\right) c_{i, j, k+1}+\left(6 B_{3}+D_{3}\right) c_{i, j, k-1}-B_{3} c_{i, j, k-2}
\end{aligned}
$$

and

$$
B_{1}:=\frac{u}{6 \Delta x}, \quad B_{2}:=\frac{v}{6 \Delta y}, \quad B_{3}:=\frac{w}{6 \Delta z}, \quad D_{1}:=\frac{\varepsilon_{x}}{(\Delta x)^{2}}, \quad D_{2}:=\frac{\varepsilon_{y}}{(\Delta y)^{2}}, \quad D_{3}:=\frac{\varepsilon_{z}}{(\Delta z)^{2}} .
$$

Here $c_{i, j, k}$ refers to the $m$-dimensional vector of concentrations $c_{\mu}$ at the grid point $P_{i j k}$.

\subsection{The Radau discretization}

Next we consider the approach in which the underlying implicit time discretization formula is of Runge-Kutta type. As an example, we will discuss the third-order, L-stable, 2-stage Radau IIA method. Then, in each step, a system has to be solved, the dimension of which is twice as large as the IVP dimension (cf. (1.4a) and (1.4b)). To avoid the increase in the linear algebra work, the Runge-Kutta matrix appearing in the modified Newton method will be approximated by a diagonal matrix $D$ or a triangular matrix $T$. This approach was followed in [5,7] to construct parallel iteration methods for solving stiff ODEs with Runge-Kutta methods. For higher-stage Runge-Kutta methods (say 4 or more stages), the "triangular" approach is to be preferred to the "diagonal" approach, in spite of its more complicated implementation requiring the use of Butcher transformations (cf. [7]). However, for the 2-stage Radau method the "diagonal" approach is clearly more efficient. Thus, approximating the Runge-Kutta matrix by the matrix $D=\operatorname{diag}\left(\alpha_{1}, \alpha_{2}\right)$, we obtain the iteration method

$$
\begin{aligned}
\boldsymbol{A}^{(\nu)}=\boldsymbol{A}^{(\nu-1)}-J_{1}^{-1} \boldsymbol{R}_{1}\left(t_{n+1 / 3}, t_{n+1}, \boldsymbol{A}^{(\nu-1)}, \boldsymbol{C}^{(\nu-1)}\right), & J_{1}:=I-\alpha_{1} \Delta t \frac{\partial \boldsymbol{F}}{\partial \boldsymbol{C}} \\
\boldsymbol{C}^{(\nu)}=\boldsymbol{C}^{(\nu-1)}-J_{2}^{-1} \boldsymbol{R}_{2}\left(t_{n+1 / 3}, t_{n+1}, \boldsymbol{A}^{(\nu-1)}, \boldsymbol{C}^{(\nu-1)}\right), & J_{2}:=I-\alpha_{2} \Delta t \frac{\partial \boldsymbol{F}}{\partial \boldsymbol{C}} \\
\quad \nu=1,2, \ldots, &
\end{aligned}
$$

where $\partial F / \partial C=\partial(H+G) / \partial C$ is again the Jacobian. Notice that both equations are of IVP dimension and, moreover, the new iterates $\boldsymbol{A}^{(\nu)}$ and $\boldsymbol{C}^{(\nu)}$ can be solved in parallel. In fact, these equations are of the same type as the modified Newton iteration (2.3) for the BDF method.

In [5] it was shown that for stiff ODEs iteration methods of the type (2.7) possess good convergence properties if the (extremely) stiff components are optimally damped. For the Radau IIA method this leads to

$$
\alpha_{1}=(4-\sqrt{6}) / 6, \quad \alpha_{2}=(4+\sqrt{6}) / 10 .
$$

In Section 3.2 we will argue that (2.8) is also a plausible choice in the present context. 
Proceeding as in the previous section, the scheme (2.7) will be further simplified by the Approximate Factorization approach to obtain

$$
\begin{aligned}
& \left(I-\alpha_{1} \Delta t X\right)\left(I-\alpha_{1} \Delta t Y\right)\left(I-\alpha_{1} \Delta t Z\right)\left(\boldsymbol{A}^{(\nu)}-\boldsymbol{A}^{(\nu-1)}\right) \\
& \quad=-\boldsymbol{R}_{1}\left(t_{n+1 / 3}, t_{n+1}, \boldsymbol{A}^{(\nu-1)}, \boldsymbol{C}^{(\nu-1)}\right), \\
& \left(I-\alpha_{2} \Delta t X\right)\left(I-\alpha_{2} \Delta t Y\right)\left(I-\alpha_{2} \Delta t Z\right)\left(\boldsymbol{C}^{(\nu)}-\boldsymbol{C}^{(\nu-1)}\right) \\
& \quad=-\boldsymbol{R}_{2}\left(t_{n+1 / 3}, t_{n+1}, \boldsymbol{A}^{(\nu-1)}, \boldsymbol{C}^{(\nu-1)}\right),
\end{aligned}
$$

where $\nu=1,2, \ldots$, and the matrices $X, Y$ and $Z$ are defined in (1.2b). Similar to the BDF-based method (cf. (2.5)), each iteration requires the successive solution of three linear systems; however, this can be done in parallel for (2.9a) and (2.9b). In this connection, we remark that in the "Gauss-Seidel" versions of (2.9), where in (2.9b) $\boldsymbol{A}^{(\nu-1)}$ is replaced by $\boldsymbol{A}^{(\nu)}$ (or, alternatively $\boldsymbol{C}^{(\nu-1)}$ is replaced by $\boldsymbol{C}^{(\nu)}$ in (2.9a)), these linear systems cannot be solved concurrently anymore. Moreover, numerical experiments reveal that for a given number of iterations, the accuracy is only slightly improved (see Section 4). Hence, we may conclude that the "Jacobi" version (2.9) is preferable to a "Gauss-Seidel" version.

\section{Convergence and stability analysis}

In the convergence and stability analysis of the iteration methods (2.5) and (2.9), we distinguish two situations: (i) iteration until convergence, and (ii) the execution of a fixed, usually small number of iterations. In the first case, we may confine our considerations to an analysis of convergence, because the stability properties of the iterated methods are those of the underlying (highly stable) BDF and Radau methods. However, if only a few iterations are performed, for instance, for reasons of efficiency, then there is no need for a convergence analysis. Instead, we should consider the order of accuracy and the stability properties after a finite number of iterations.

In this paper, we confine our considerations to a convergence analysis of (2.5) and (2.9). For a detailed convergence and stability analysis of a wide class of numerical integration methods using iteration methods based on approximate factorization, we refer to the forthcoming paper [2].

Our convergence analysis is based on a normal mode analysis using the "frozen coefficients" approach, that is, the quantities $u, v, w, \varepsilon_{x}, \varepsilon_{y}, \varepsilon_{z}$ are assumed to be constant. Again, we discuss the $\mathrm{BDF}$ and Radau discretizations separately.

\subsection{The BDF discretization}

Let us define the iteration error $\boldsymbol{e}^{(\nu)}:=\boldsymbol{C}^{(\nu)}-\boldsymbol{C}_{n+1}$. Then

$$
\begin{aligned}
(I-\alpha \Delta t X)(I-\alpha \Delta t Y)(I-\alpha \Delta t Z)\left(e^{(\nu)}-\boldsymbol{e}^{(\nu-1)}\right) \\
\quad=-\left(\boldsymbol{R}\left(t_{n+1}, \boldsymbol{C}^{(\nu-1)}\right)-\boldsymbol{R}\left(t_{n+1}, \boldsymbol{C}_{n+1}\right)\right) \\
\quad \approx-\left(I-\alpha \Delta t \frac{\partial \boldsymbol{H}}{\partial \boldsymbol{C}}-\alpha \Delta t \frac{\partial \boldsymbol{G}}{\partial \boldsymbol{C}}\right) \boldsymbol{e}^{(\nu-1)} .
\end{aligned}
$$


observing that $\partial H / \partial C=X+Y+Z$, where the matrices $X, Y$ and $Z$ are defined according to b), we obtain the error recursion

$$
\begin{aligned}
\boldsymbol{e}^{(\nu)}= & M \boldsymbol{e}^{(\nu-1)} \\
M:= & I-(I-\alpha \Delta t Z)^{-1}(I-\alpha \Delta t Y)^{-1}(I-\alpha \Delta t X)^{-1} \\
& \times\left(I-\alpha \Delta t(X+Y+Z)-\alpha \Delta t \frac{\partial G}{\partial C}\right) .
\end{aligned}
$$

have convergence if the eigenvalues $\mu$ of $M$ are within the unit circle. Since the reaction terms nonstiff, we ignore the term $\alpha \Delta t \partial G / \partial C$, so that a normal mode analysis can be applied. Let

$$
\begin{aligned}
& M \exp \left(\mathrm{i}\left(\omega_{1} x+\omega_{2} y+\omega_{3} z\right)\right)=\mu \exp \left(\mathrm{i}\left(\omega_{1} x+\omega_{2} y+\omega_{3} z\right)\right), \\
& \theta_{1}:=\omega_{1} \Delta x, \quad \theta_{2}:=\omega_{2} \Delta y, \quad \theta_{3}:=\omega_{3} \Delta z .
\end{aligned}
$$

$\mathrm{n}$ it follows from (3.2) and (2.6) that

$$
\mu=1-\frac{1-\alpha \Delta t\left(\lambda_{1}\left(\theta_{1}\right)+\lambda_{2}\left(\theta_{2}\right)+\lambda_{3}\left(\theta_{3}\right)\right)}{\left(1-\alpha \Delta t \lambda_{1}\left(\theta_{1}\right)\right)\left(1-\alpha \Delta t \lambda_{2}\left(\theta_{2}\right)\right)\left(1-\alpha \Delta t \lambda_{3}\left(\theta_{3}\right)\right)}, \quad\left|\theta_{j}\right| \leqslant \pi, \quad j=1,2,3,
$$

re the $\lambda_{j}\left(\theta_{j}\right)$ represent the eigenvalues of the matrices $X, Y$ and $Z$, i.e.,

$$
\begin{aligned}
& \lambda_{j}\left(\theta_{j}\right)=-B_{j} \gamma\left(\theta_{j}\right)+D_{j} \delta\left(\theta_{j}\right), \\
& \gamma(\theta):=2 \mathrm{e}^{\mathrm{i} \theta}+3-6 \mathrm{e}^{-\mathrm{i} \theta}+\mathrm{e}^{-2 \mathrm{i} \theta}=2(\cos (\theta)-1)^{2}+2 \mathrm{i}(4-\cos (\theta)) \sin (\theta), \\
& \delta(\theta):=\mathrm{e}^{-\mathrm{i} \theta}-2+\mathrm{e}^{\mathrm{i} \theta}=2(\cos (\theta)-1) .
\end{aligned}
$$

alling that the magnitude of $B_{3}$ is usually much larger than that of $B_{1}$ and $B_{2}$, we consider the ting case where $B_{3}=\infty$ (i.e., $\Delta z \rightarrow 0$ and $w \neq 0$ ), to obtain

$$
\begin{aligned}
& \mu\left(\theta_{1}, \theta_{2}, \theta_{3}\right)=1-\frac{1}{\left(1-\alpha \Delta t \lambda_{1}\left(\theta_{1}\right)\right)\left(1-\alpha \Delta t \lambda_{2}\left(\theta_{2}\right)\right)}, \\
& \quad \theta_{3} \neq 0, \quad\left|\theta_{j}\right| \leqslant \pi, \quad j=1,2, \\
& \mu\left(\theta_{1}, \theta_{2}, 0\right)=1-\frac{1-\alpha \Delta t\left(\lambda_{1}\left(\theta_{1}\right)+\lambda_{2}\left(\theta_{2}\right)\right)}{\left(1-\alpha \Delta t \lambda_{1}\left(\theta_{1}\right)\right)\left(1-\alpha \Delta t \lambda_{2}\left(\theta_{2}\right)\right)}, \quad\left|\theta_{j}\right| \leqslant \pi, \quad j=1,2 .
\end{aligned}
$$

In straightforwardly be verified that these expressions are on the open unit disk if

$$
\begin{aligned}
& \left({ }_{x} \Delta t\right)^{2} \operatorname{Re}\left(\lambda_{1}\left(\theta_{1}\right) \lambda_{2}\left(\theta_{2}\right)\right)-\alpha \Delta \Delta t \operatorname{Re}\left(\lambda_{1}\left(\theta_{1}\right)+\lambda_{2}\left(\theta_{2}\right)\right)+\frac{1}{2}>0, \\
& { } \Delta t \operatorname{Re}\left(\lambda_{j}\left(\theta_{j}\right)\right)<\frac{1}{2},
\end{aligned}
$$

re $\left|\theta_{j}\right| \leqslant \pi, j=1,2$. The second inequality is always satisfied (see (3.4)) and the first inequality ttisfied if $\operatorname{Re}\left(\lambda_{1}\left(\theta_{1}\right) \lambda_{2}\left(\theta_{2}\right)\right) \geqslant 0$. If $\operatorname{Re}\left(\lambda_{1}\left(\theta_{1}\right) \lambda_{2}\left(\theta_{2}\right)\right)<0$, then we obtain an upper bound for $\Delta t$. vever, this upper bound is positive and certainly greater than

$$
\left[-2 \alpha^{2} \min \left\{\operatorname{Re}\left(\lambda_{1}\left(\theta_{1}\right) \lambda_{2}\left(\theta_{2}\right)\right)\right\}\right]^{-1 / 2} \text {. }
$$


Table 1

CFL numbers and maximal convergent stepsizes for the BDF discretization

\begin{tabular}{llllllllllll}
\hline$q=$ & $\infty$ & 100 & 50 & 20 & 10 & 5 & 2 & 1 & 0.5 & 0.1 & 0 \\
\hline$Q=$ & $\infty$ & 99 & 50 & 21 & 11.5 & 6.9 & 4.5 & 4.1 & 4.4 & 7.9 & $\infty$ \\
$\Delta t B_{1} \leqslant$ & 0.18 & 0.16 & 0.16 & 0.16 & 0.16 & 0.16 & 0.18 & 0.22 & 0.29 & 0.62 & $\infty$ \\
\hline
\end{tabular}

Since

$$
\left|\operatorname{Re}\left(\lambda_{1}\left(\theta_{1}\right) \lambda_{2}\left(\theta_{2}\right)\right)\right| \leqslant\left|\lambda_{1}\left(\theta_{1}\right) \lambda_{2}\left(\theta_{2}\right)\right| \leqslant\left(\gamma_{0} B_{1}+4 D_{1}\right)\left(\gamma_{0} B_{2}+4 D_{2}\right)
$$

where $\gamma_{0}:=\max \{|\gamma(\theta)|\}$, we conclude that a sufficient convergence condition is

$$
\Delta t \leqslant \frac{1}{\alpha \sqrt{2\left(\gamma_{0} B_{1}+4 D_{1}\right)\left(\gamma_{0} B_{2}+4 D_{2}\right)}}, \quad \gamma_{0}:=\max \{|\gamma(\theta)|\}=9 .
$$

Thus, we have the following convergence theorem:

Theorem 3.1. If $w \neq 0$ and $\Delta z \rightarrow 0$, then a sufficient condition for convergence is given by (3.7).

Remark 3.1. In the case where $w=0$ (i.e., $B_{3}=0$ ) and a sufficiently small vertical diffusion coefficient, the eigenvalues $\mu$ can be approximated by $(3.5 \mathrm{~b})$. We already showed that $(3.5 \mathrm{~b})$ assumes values within the unit circle if (3.6b) is satisfied, so that we have unconditional convergence if $B_{3}=0$.

In order to obtain some quantitative information on the size of the CFL numbers and the maximal convergent stepsize, consider a pure advection model problem $\left\{B_{1}=B_{2}, B_{3}=q B_{1}, \quad D_{j}=0\right\}$. Defining the quantity $b=\alpha B_{1} \Delta t$, we obtain

$$
\mu\left(\theta_{1}, \theta_{2}, \theta_{3}\right)=1-\frac{1+b \gamma\left(\theta_{1}\right)+b \gamma\left(\theta_{2}\right)+q b \gamma\left(\theta_{3}\right)}{\left(1+b \gamma\left(\theta_{1}\right)\right)\left(1+b \gamma\left(\theta_{2}\right)\right)\left(1+q b \gamma\left(\theta_{3}\right)\right)}, \quad\left|\theta_{j}\right| \leqslant \pi, \quad j=1,2,3 .
$$

For this model problem, the CFL number is given by $Q=6(2+q) B_{1} \Delta t=6(2+q) b \alpha^{-1}$, so that for a given $q$, the maximal convergent CFL number can be computed by finding the largest value of $b$ for which $\mu\left(\theta_{1}, \theta_{2}, \theta_{3}\right)$ remains on the open unit disk when $\left(\theta_{1}, \theta_{2}, \theta_{3}\right)$ runs through the frequency space. In Table 1 we have listed the CFL number for a sequence of $q$-values. In addition, we listed the maximal values of $\Delta t B_{1}$, i.e., $\Delta t B_{1}=Q(6(2+q))^{-1}$.

Realistic values for $B_{1}$ and $B_{2}$ in a shallow water transport problem are $B_{1}=B_{2}=1 / 6000$ (corresponding to, for example, $|u|=|v|=1 \mathrm{~m} \mathrm{sec}^{-1}$ and $\Delta x=\Delta y=1000 \mathrm{~m}$ ). Then, the most critical $q$-value still allows for a time step of approximately 16 minutes.

It is of interest to apply the sufficient condition (3.7) of Theorem 3.1 to the above model problem $\left\{B_{1}=B_{2}, D_{j}=0\right\}$. This yields $\Delta t B_{1} \leqslant 0.12$. Since $w \neq 0$ and $\Delta z \rightarrow 0$ implies that $q=x$, this sufficient condition should be compared with the true condition $\Delta t B_{1} \leqslant 0.18$ of Table 1. Thus, condition (3.7) prescribes stepsizes that are a factor $2 / 3$ smaller than really necessary.

Furthermore, it is of interest to know which frequencies are responsible for the limitation of the time step. To get an impression, we again use the pure advection problem $\left\{B_{1}=B_{2}, B_{3}=q B_{1}, D_{j}=0\right\}$, apply the maximal value for $\Delta t$ and we consider the $|\mu|$-values as a function of $\theta_{1}, \theta_{2}$ and $\theta_{3}$. In Fig. 1 $|\mu|$-values are plotted for $q=100$. In this plot, the horizontal axes contain $\theta_{1}$ - and $\theta_{3}$-values; $\theta_{2}$ has been omitted since we observed that the maximal $\Delta t$ was always obtained for $\theta_{1}=\theta_{2}$. We see that $|\mu|$ 


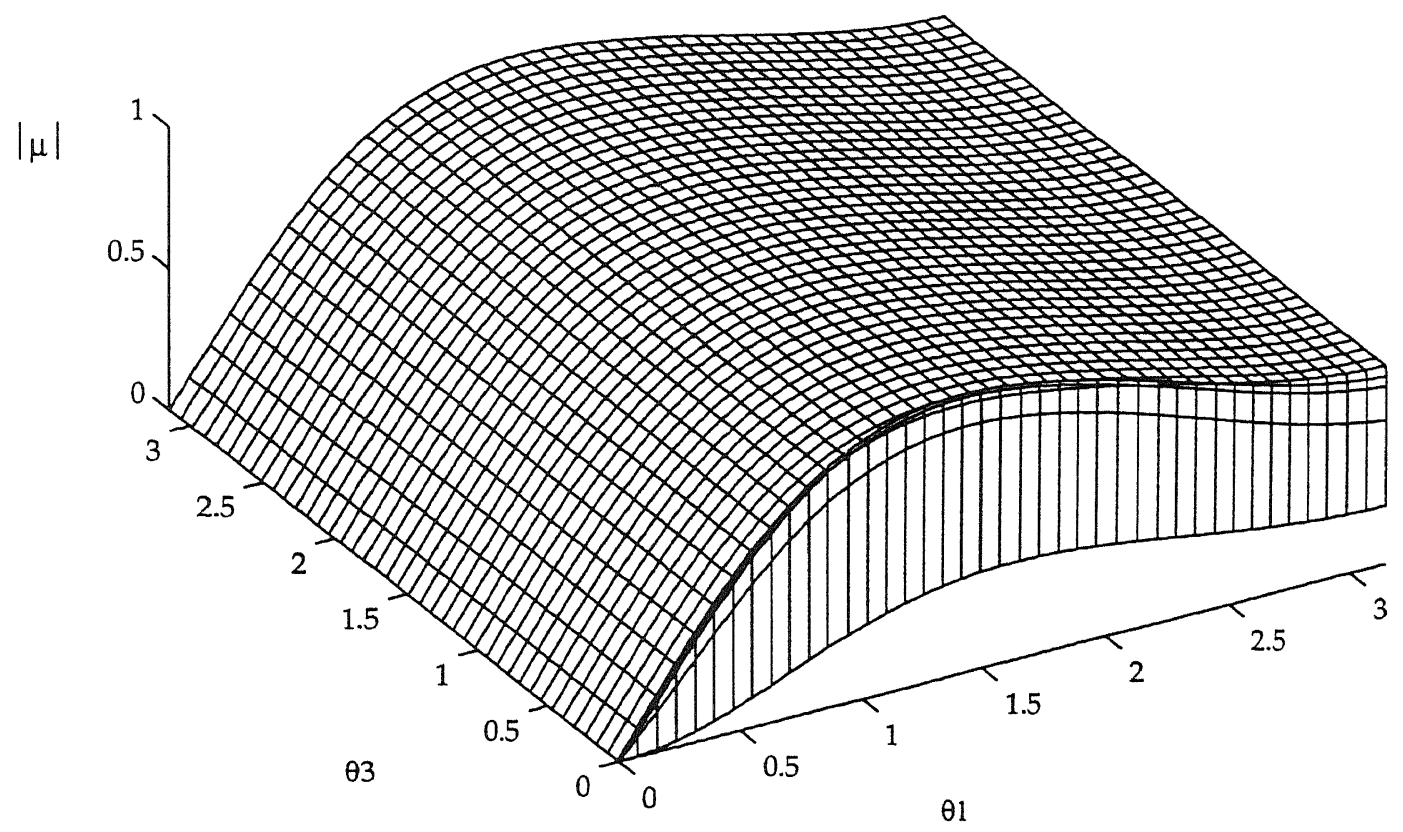

Fig. 1. $|\mu|$-values (3.3) for $q=100$.

does not critically depend on $\theta_{3}$, except for $\theta_{3}=0$. In that case, the expression for $\mu$ reduces to (3.5b for which it was shown that there is no restriction on the time step. Furthermore, we observe $t^{1}$ the low frequencies in the $\theta_{1}$-direction are perfectly damped and the highest "horizontal" frequenc $\left(\theta_{1} \approx \pi\right)$ are treated satisfactorily. However, for small $q$, the damping of these frequencies tends one. Finally, this plot clearly indicates that the mid-range in the frequency space causes the condition on the time step.

\subsection{The Radau discretization}

Proceeding as for the BDF discretization, we obtain for the iteration errors $\boldsymbol{e}_{1}^{(\nu)}:=\boldsymbol{A}^{(\nu)}-\boldsymbol{A}_{n+1 / 3}$ and $e_{2}^{(\nu)}:=C^{(\nu)}-C_{n+1}$ the error recursion

$$
\left(\begin{array}{c}
e_{1}^{(\nu)} \\
e_{2}^{(\nu)}
\end{array}\right) \approx M\left(\begin{array}{c}
e_{1}^{(\nu-1)} \\
e_{2}^{(\nu-1)}
\end{array}\right), \quad M:=\left(\begin{array}{cc}
I-P_{1}^{-1}\left(I-\frac{5}{12} \Delta t S\right) & -\frac{1}{12} \Delta t P_{1}^{-1} S \\
\frac{3}{4} \Delta t P_{2}^{-1} S & I-P_{2}^{-1}\left(I-\frac{1}{4} \Delta t S\right)
\end{array}\right),
$$

where

$$
P_{j}:=\left(I-\alpha_{j} \Delta t X\right)\left(I-\alpha_{j} \Delta t Y\right)\left(I-\alpha_{j} \Delta t Z\right), \quad S:=X+Y+Z+\frac{\partial \boldsymbol{G}}{\partial \boldsymbol{C}}, \quad j=1,2 .
$$

Ignoring the reaction terms and applying a normal mode analysis, we see that the eigenvalues of $M$ are given by the eigenvalues of the matrix 
Table 2

CFL numbers and maximal convergent stepsizes for the Radau discretization

\begin{tabular}{llllllllllll}
\hline$q=$ & $\infty$ & 100 & 50 & 20 & 10 & 5 & 2 & 1 & 0.5 & 0.1 & 0 \\
\hline$Q=$ & $\infty$ & 107 & 55 & 23 & 12.6 & 7.5 & 4.8 & 4.4 & 4.8 & 8.7 & $\infty$ \\
$\Delta t B_{1} \leqslant$ & 0.19 & 0.18 & 0.17 & 0.17 & 0.17 & 0.18 & 0.20 & 0.24 & 0.31 & 0.69 & $\infty$ \\
\hline
\end{tabular}

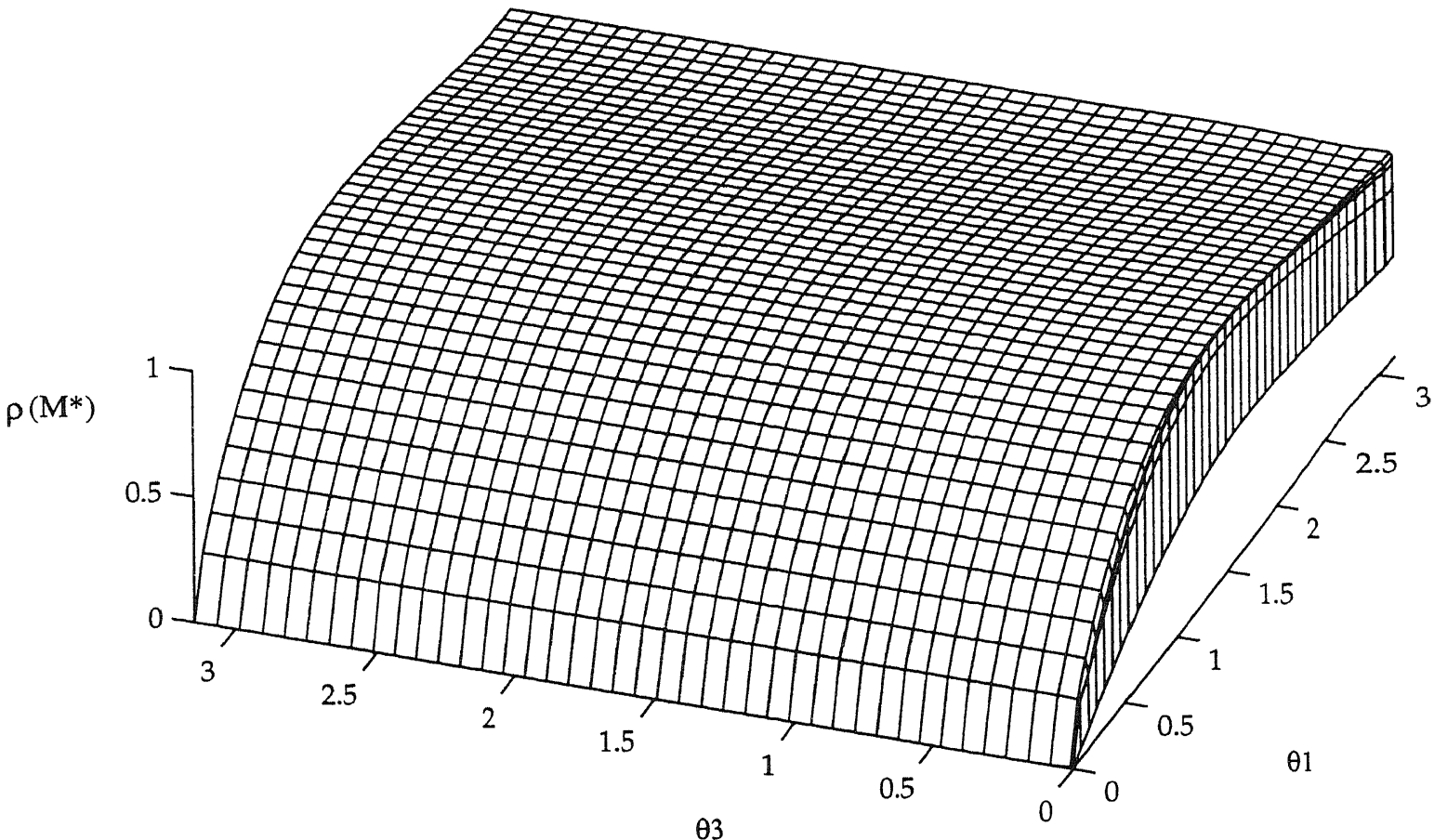

$\theta 3$

Fig. 2. Spectral radius of $M^{*}$ (cf. (3.9)) with $q=100$ and $\alpha_{1}, \alpha_{2}$ according to (2.8).

$$
M^{*}:=\left(\begin{array}{cc}
1-\frac{1-\frac{5}{12} \Delta t \lambda(S)}{\lambda\left(P_{1}\right)} & -\frac{1}{12} \Delta t \frac{\lambda(S)}{\lambda\left(P_{1}\right)} \\
\frac{3}{4} \Delta t \frac{\lambda(S)}{\lambda\left(P_{2}\right)} & 1-\frac{1-\frac{1}{4} \Delta t \lambda(S)}{\lambda\left(P_{2}\right)}
\end{array}\right),
$$

where

$$
\begin{aligned}
& \lambda(S)=\lambda_{1}\left(\theta_{1}\right)+\lambda_{2}\left(\theta_{2}\right)+\lambda_{3}\left(\theta_{3}\right), \\
& \lambda\left(P_{j}\right)=\left(1-\alpha_{j} \Delta t \lambda_{1}\left(\theta_{1}\right)\right)\left(1-\alpha_{j} \Delta t \lambda_{2}\left(\theta_{2}\right)\right)\left(1-\alpha_{j} \Delta t \lambda_{3}\left(\theta_{3}\right)\right), \quad j=1,2,
\end{aligned}
$$

and where $\lambda_{j}\left(\theta_{j}\right)$ is defined in (3.4). Convergence requires that the eigenvalues $\mu$ of $M^{*}$ are within the unit circle. Table 2 represents the analogue of Table 1 for the advection-dominated model problem $\left\{B_{1}=B_{2}, B_{3}=q B_{1}, D_{j}=0\right\}$. A comparison of these tables reveals that the Radau discretization imposes slightly less restrictive convergence conditions than the BDF discretization. 


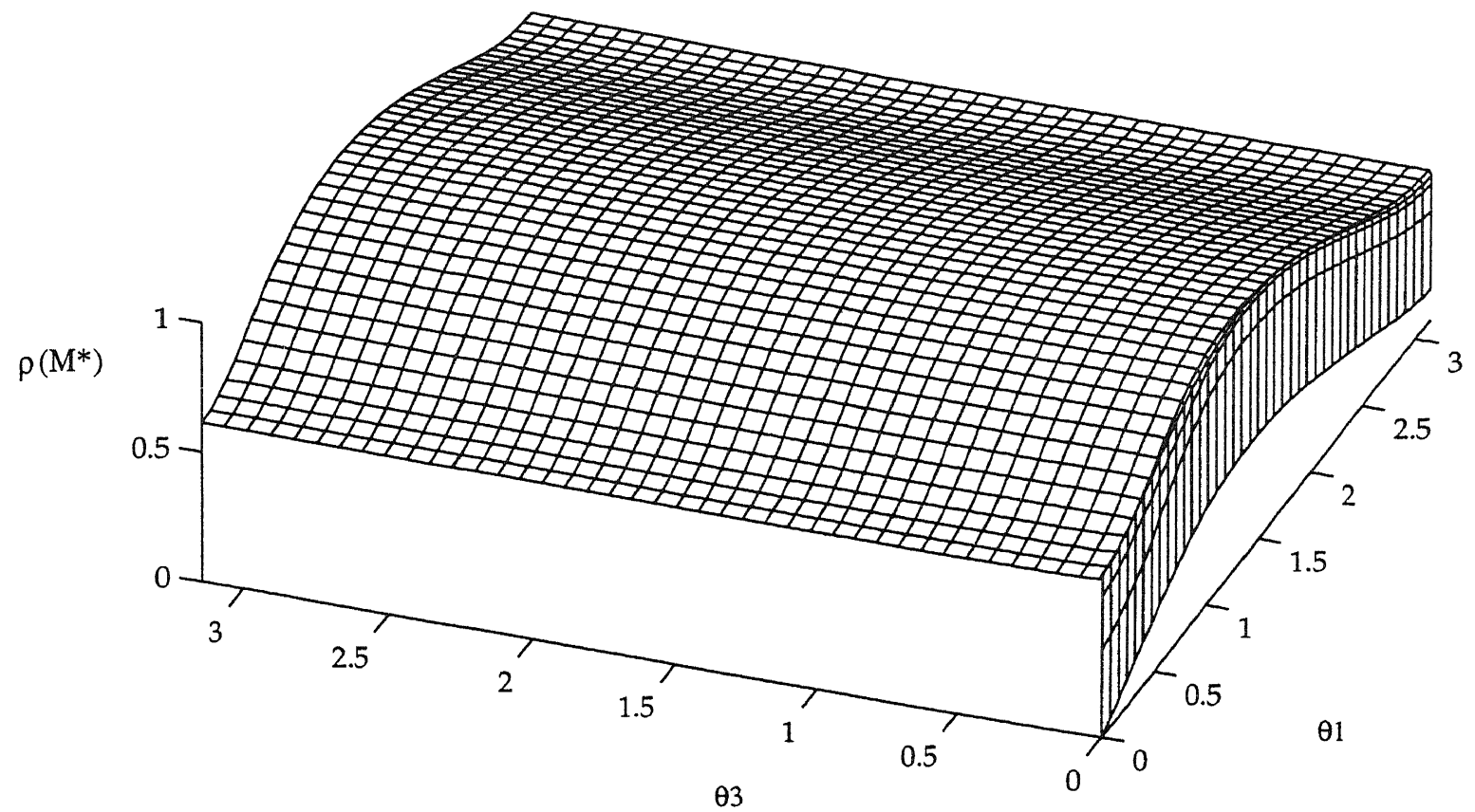

Fig. 3. Spectral radius of $M^{*}$ (cf. (3.9)) with $q=100$ and $\alpha_{1}=0.19, \alpha_{2}=0.40$.

Similar to the preceding section, we want to know which frequencies give rise to the critical time step with respect to convergence. To that end, we present in Fig. 2 a plot of the spectral radius of the matrix $M^{*}$ as a function of the spatial frequencies $\theta_{1}$ and $\theta_{3}$. Here we again used the above model problem with $q=100$ and the maximally allowed time step. We observe a similar behaviour as for the BDF case.

Next, we discuss the choice of the parameters $\alpha_{1}$ and $\alpha_{2}$, as introduced in Section 2.2. As explained there, these parameters were selected on the basis of previous research of related iteration methods to solve stiff ODEs. It is, however, not a priori evident that (2.8) is also the best choice in the present application. To see whether there exist values that allow for a larger time step, we performed a numerical search in the $\left(\alpha_{1}, \alpha_{2}\right)$-parameter space (again for the model problem with $q=100$ ). It turned out that $\alpha_{1}=0.19, \alpha_{2}=0.40$ are the optimal values for the Radau IIA method. These values give rise to a bound of 0.22 for $\Delta t B_{1}$, which is an increase of $\approx 20 \%$ compared with the value 0.18 as listed in Table 2. There is, however, a price to be paid in taking these optimal $\alpha$-values. As we see from Fig. 3 (where we plotted the spectral radius of $M^{*}$ for $\alpha_{1}=0.19, \alpha_{2}=0.40, q=100$, and the largest possible time step), the low frequency components in the $\theta_{1}$ direction are damped with a factor close to 0.6. This is an undesirable situation and indeed, using these $\alpha$-values, we observed a reduced convergence behaviour in the numerical test problem, described in Section 4. The poor convergence of the low-frequency components is easily understood by analyzing the matrix $M^{*}$ in (3.9) for $\theta_{1}=\theta_{2}=0$.

Then this matrix reduces to

$$
M^{*}=z(I-z D)^{-1}(A-D),
$$


where $z:=\Delta t \lambda_{3}\left(\theta_{3}\right), D:=\operatorname{diag}\left(\alpha_{1}, \alpha_{2}\right)$ and $A$ is the Radau matrix. Since $|z|=\left|B_{3} \Delta t \gamma_{3}\left(\theta_{3}\right)\right|$ will take large values in many practical situations, the matrix $M^{*}$ behaves like $I-D^{-1} A$ (indeed, the "optimal" choice $\left(\alpha_{1}, \alpha_{2}\right)=(0.19,0.40)$ leads to eigenvalues $\approx 0.61$ for this matrix). In [5], we studied exactly the same iteration matrix $M^{*}$ as given in $\left(3.9^{\prime}\right)$; the choice (2.8) resulted from requiring that $I-D^{-1} A$ possesses a zero spectrum. Therefore, we also adopt this choice in the present application, in spite of the fact that the maximally allowed time step in order to obtain convergence for the mid-range frequencies is slightly reduced.

\section{Numerical experiments}

In this paper, we have confined our attention to a relatively simple test problem. In future publications, numerical results for more complex transport problems will be reported. Our test problem is defined by

$$
\begin{aligned}
& \frac{\partial c_{1}}{\partial t}+U \cdot \nabla c_{1}=\varepsilon \Delta c_{1}+g_{1}(t, x, y, z)-k_{1} c_{1} c_{2}, \\
& \frac{\partial c_{2}}{\partial t}+U \cdot \nabla c_{2}=\varepsilon \Delta c_{2}+g_{2}(t, x, y, z)-k_{1} c_{1}+k_{2}\left(1-c_{2}\right), \\
& \quad 0 \leqslant x, y \leqslant L_{h}, \quad-L_{v} \leqslant z \leqslant 0, \quad 0 \leqslant t \leqslant T
\end{aligned}
$$

where $U=(u, v, w)$ denotes the divergence free velocity field, given in analytical form (see [1])

$$
\begin{aligned}
& u(t, x, y, z)=\left\{\widetilde{y}+3\left(\widetilde{z}+\frac{1}{2}\right)\left[\left(\widetilde{x}-\frac{1}{2}\right)^{2}+\left(\widetilde{y}-\frac{1}{2}\right)^{2}-p^{2}\right]\right\} d(t), \\
& v(t, x, y, z)=\left\{-\widetilde{x}+3\left(\widetilde{z}+\frac{1}{2}\right)\left[\left(\widetilde{x}-\frac{1}{2}\right)^{2}+\left(\widetilde{y}-\frac{1}{2}\right)^{2}-p^{2}\right]\right\} d(t), \\
& w(t, x, y, z)=-3 L_{v} \widetilde{z}(\widetilde{z}+1)\left\{\left(\widetilde{x}-\frac{1}{2}\right) / L_{h}+\left(\widetilde{y}-\frac{1}{2}\right) / L_{h}\right\} d(t),
\end{aligned}
$$

where we used the scaled co-ordinates $\widetilde{x}:=x / L_{h}, \widetilde{y}:=y / L_{h}, \widetilde{z}:=z / L_{v}$, and $p=\frac{1}{3}$ and $d(t)=$ $\cos \left(2 \pi t / T_{p}\right)$. The Dirichlet boundary conditions, the initial condition and the functions $g_{1}$ and $g_{2}$ are chosen in accordance with the prescribed analytical solution, which is of the form

$$
c_{\mu}(t, x, y, z)=\exp \left\{\tilde{z} / \mu-f_{\mu}(t)-\gamma_{\mu}\left[(\widetilde{x}-r(t))^{2}+(\widetilde{y}-s(t))^{2}\right]\right\}, \quad \mu=1,2,
$$

with

$$
f_{2}(t)=\frac{t}{T_{b}+t}, \quad f_{1}(t)=4 f_{2}(t), \quad r(t)=\frac{2+\cos \left(2 \pi t / T_{p}\right)}{4}, \quad s(t)=\frac{2+\sin \left(2 \pi t / T_{p}\right)}{4} .
$$

In our experiments, we take the following values for the parameters: $L_{h}=20000, L_{v}=100$, $\varepsilon=0.5, \gamma_{1}=80, \gamma_{2}=20, T_{b}=32400$, and $T_{p}=43200$. We use two grids, respectively with $N_{x}=N_{y}=41, N_{z}=6$ (coarse grid) and $N_{x}=N_{y}=81, N_{z}=11$ (fine grid). The length of the integration interval $T=36000$. Realistic values for the reaction rate constants are: $k_{1}=k_{2}=10^{-4}$.

The accuracy is measured by

$$
\mathrm{cd}_{\mu}:=\text { minimum over all grid points }\left(-\log _{10} \mid \text { absolute error for } c_{\mu} \mid\right), \quad \mu=1,2 .
$$


Table 3

$\mathrm{cd}_{1} / \mathrm{cd}_{2}$-values obtained by the RBWLH method [4]. $N=$ the number of time steps $(\Delta t=T / N)$; an unstable behaviour is indicated by an $*$

\begin{tabular}{lcccccccccc}
\hline Spatial grid & $N=10$ & $N=20$ & $N=35$ & $N=70$ & $N=140$ & $N=280$ & $N=560$ & $\cdots$ & $N \rightarrow \infty$ & $\mathrm{CPU} /$ step \\
\hline Grid $_{\text {coarse }}$ & $*$ & $*$ & $2.8 / 1.8$ & $3.5 / 2.4$ & $3.8 / 3.0$ & $3.8 / 3.6$ & $3.8 / 4.2$ & $\cdots$ & $3.8 / 4.3$ & 0.062 \\
Grid $_{\text {fine }}$ & $*$ & $*$ & $*$ & $2.9 / 1.8$ & $3.6 / 2.5$ & $4.3 / 3.0$ & $4.8 / 3.7$ & $\cdots$ & $4.8 / 5.2$ & 0.28 \\
\hline
\end{tabular}

Table 4

$\mathrm{cd}_{1} / \mathrm{cd}_{2}$-values obtained by the BDF method $\{(1.3),(2.5)\} . N=$ the number of time steps $(\Delta t=T / N)$

\begin{tabular}{lccccccccccc}
\hline Spatial grid & $\nu$ & $N=10$ & $N=20$ & $N=35$ & $N=70$ & $N=140$ & $N=280$ & $N=560$ & $\cdots$ & $N \rightarrow \infty$ & CPU/step \\
\hline Grid $_{\text {coarse }}$ & 2 & $1.7 / 1.4$ & $2.0 / 1.8$ & $2.4 / 2.2$ & $2.9 / 2.8$ & $3.4 / 3.4$ & $3.7 / 3.9$ & $3.8 / 4.1$ & $\cdots$ & $3.8 / 4.3$ & 0.024 \\
Grid $_{\text {fine }}$ & 2 & $1.6 / 1.4$ & $2.0 / 1.8$ & $2.4 / 2.2$ & $2.9 / 2.8$ & $3.5 / 3.4$ & $4.1 / 4.0$ & $4.5 / 4.5$ & $\cdots$ & $4.8 / 5.2$ & 0.13 \\
\hline
\end{tabular}

Table 5

$\mathrm{cd}_{1} / \mathrm{cd}_{2}$-values obtained by the Radau method $\{(1.4),(2.8),(2.9)\} . N=$ the number of time steps $(\Delta t=T / N)$

\begin{tabular}{lccccccccccc}
\hline Spatial grid & $\nu$ & $N=10$ & $N=20$ & $N=35$ & $N=70$ & $N=140$ & $N=280$ & $N=560$ & $\cdots$ & $N \rightarrow \infty$ & CPU/step \\
\hline Grid $_{\text {coarse }}$ & 3 & $2.1 / 2.1$ & $3.1 / 3.0$ & $3.7 / 3.6$ & $3.8 / 4.2$ & $3.8 / 4.3$ & & $\cdots$ & $3.8 / 4.3$ & 0.064 \\
Grid $_{\text {fine }}$ & 3 & $2.0 / 2.0$ & $2.8 / 2.6$ & $3.5 / 3.2$ & $4.2 / 4.0$ & $4.7 / 4.6$ & $4.8 / 5.1$ & $\cdots$ & $4.8 / 5.2$ & 0.33 \\
\hline
\end{tabular}

The iteration processes in the BDF and Radau methods $\{(1.3),(2.5)\}$ and $\{(1.4),(2.8),(2.9)\}$ are applied with $\nu$ iterations in each step, where $\nu$ is specified in the tables of results. These values are more or less optimal with respect to efficiency and have been obtained experimentally. It turns out that BDF needs less iterations than the Radau method. This can be explained by observing that in the BDF case, each iteration damps the nonstiff error components by a factor $\mathrm{O}\left((\Delta t)^{2}\right)$, because the eigenvalues $\mu$ defined by (3.3) are $\mathrm{O}\left((\Delta t)^{2}\right)$. In the Radau case, it can be deduced from (3.9) that its eigenvalues are only $\mathrm{O}(\Delta t)$.

Since the Jacobian matrices $\partial \boldsymbol{H} / \partial \boldsymbol{C}$ only depend on $t$ (and not on $\boldsymbol{C}$ ), they are evaluated at the new time levels. To start the iteration, we use the trivial prediction, i.e., $C^{(0)}:=C_{n}$ in BDF and $\boldsymbol{A}^{(0)}:=\boldsymbol{C}^{(0)}:=\boldsymbol{C}_{n}$ in the Radau method. It turns out that this choice for $\boldsymbol{C}^{(0)}$ is more robust than using a (more accurate) extrapolation formula. In addition to these iteration methods, we apply the RBWLH method developed in [6] (see also [4]). For various values of $\Delta t$, the cd-values and the CPU time per step are given in Tables 3, 4 and 5. These CPU times are obtained when running the codes in vector mode on one processor of a CRAY C98/4256. Evidently, the new methods allow considerably larger stepsizes than the RBWLH method; even for time steps as large as 1 hour, the new methods are able to produce results of resonable accuracy. Another nice property of the new methods is that they do not show the Du Fort-Frankel inconsistency as observed in the RBWLH method. By this we mean that, if both $\Delta t$ and the grid meshes are halved, then the accuracy of the BDF and Radau method increases proportionally. Also note that for $N \rightarrow \infty$ (that is, $\Delta t \rightarrow 0$ ) all methods show a third-order behaviour in space, as is to be expected from the upwind discretization of the advection terms, which form the most important part in the model. In case the temporal error dominates the spatial error (small $N$-values), we see that the Radau method is the most accurate one; this is obviously owing to its third-order accuracy in time. Furthermore, if we take into account that the two sets of 
linear systems for $\boldsymbol{A}^{(\nu)}$ and $\boldsymbol{C}^{(\nu)}$ in the Radau-based method (2.9) can be solved in parallel, we see that per step both the BDF and the Radau method are cheaper than the RBWLH method. Finally, we tested "Gauss-Seidel" versions of the Radau type method (2.9). As already observed at the end of Section 2.2, the accuracies listed in Table 5 are only marginally improved, so that in this application the "Jacobi" version (2.9) is a more efficient approach.

\section{References}

[1] D. Dunsbergen, Particle models for transport in three-dimensional shallow water flow, Ph.D. Thesis, Delft Technical University (1994).

[2] C. Eichler-Liebenow, P.J. van der Houwen and B.P. Sommeijer, Analysis of approximate factorization in iteration methods, in preparation.

[3] C. Hirsch, Numerical Computation of Internal and External Flows, Vol. 1: Fundamentals of Numerical Discretization (Wiley, New York, 1988).

[4] B.P. Sommeijer and J. Kok, Splitting methods for three-dimensional bio-chemical transport, Appl. Numer. Math. 21 (1996) 303-320.

[5] P.J. van der Houwen and B.P. Sommeijer, Iterated Runge-Kutta methods on parallel computers, SIAM J. Sci. Statist. Comput. 12 (1991) 1000-1028.

[6] P.J. van der Houwen and B.P. Sommeijer, Splitting methods for three-dimensional transport models with interaction terms, CWI Report NM-R9516, submitted for publication.

[7] P.J. van der Houwen and J.J.B. de Swart, Triangularly implicit iteration methods for ODE-IVP solvers, SIAM J. Sci. Comput. 18 (1996) 41-55.

[8] B. van Leer, Upwind-difference methods for aerodynamic problems governed by the Euler equations, in: B.E. Engquist, S. Osher and R.C.J. Sommerville, eds., Proceedings of the 15th AMS-SIAM Summer Seminar on Applied Mathematics, Scripps Institution of Oceanography, 1983, Lectures in Applied Mathematics 22. Part 2 (American Mathematical Society, Providence, RI, 1985) 327-336. 